International Journal of Ambient Systems and Applications (IJASA) Vol.1, No.3,september 2013

\title{
A KEY LEVEL SELECTION WITHIN HASH CHAINS FOR THE EFFICIENT ENERGY CONSUMPTION IN WSNS
}

\author{
Hyun Woo Lee ${ }^{1}$, Su Man Nam² and Tae Ho Cho ${ }^{3}$ \\ ${ }^{123}$ College of Information and Communication Engineering, Sungkyunkwan University, \\ Suwon 440-746, Republic of Korea
}

\begin{abstract}
A wireless sensor network is composed of a base station (BS) and numerous sensor nodes. The sensor nodes lack security because they operate in an open environment, such as the military. In particular, a false report injection attack captures and compromises sensor nodes. The attack then causes the compromised nodes to generate forward false reports. Owing to the false report injection attack, not only does the sensor network have a false alarm, but its limited energy is also drained. In order to defend the false report injection attack, over the past few years, several studies have been made looking for a solution to the attack. Ye et al. studied statistical en-route filtering (SEF). SEF is a method of stochastically verifying event reports in the en-route filtering phase. SEF can filter many false reports early using verification of intermediate nodes. However, because the number of keys in a sensor node is fixed by the system, the sensor network cannot control the event report verification probability depending on the circumstances of the network. Therefore, it is difficult to efficiently consume energy of the sensor network. In order to solve the problem, we propose a method which controls the event report verification probability by using a key sequence level of an event report. In the proposed method, when an intermediate node receives an event report, the node verifies the event report by comparing a key sequence level of the report and its key sequence level. Elements determining the key sequence level include the density of neighbour nodes in the sensing range of a center of stimulus $(C o S)$, the number of hops from the CoS to the BS, and the average of the key sequence level of intermediate nodes in each path. We simulated the proposed method and the SEF method to evaluate the performance in terms of energy efficiency and security. In the simulation results, the proposed method consumed an average of $7.9 \%$ less energy of the sensor nodes compared to SEF method. The number of false reports arriving at the BS of the proposed method was also less, by an average of 6.4, compared to the SEF method. Through the results, we can see that when the number of false report is large in the sensor network, the proposed method is more energy-efficient and secure than the SEF method.
\end{abstract}

\section{KEYWORDS}

Wireless sensor network, False report injection attack, Statistical en-route filtering, Energy Efficiency, Security

DOI : $10.5121 /$ ijasa.2013.1301 


\section{INTRODUCTION}

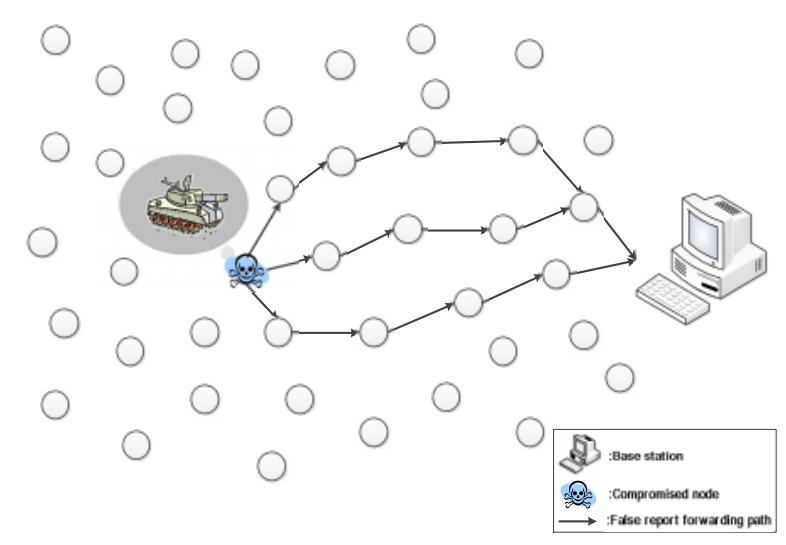

Fig. 1. The operation of a false report injection attack

Wireless sensor networks consist of a large number of sensor nodes, which include sensing, computation, and wireless communication capabilities $[1,2]$. The sensor nodes are allocated in a particular sensor field and operate each other collaboratively [1]. When an event occurs in the sensor field, the nodes sense and compute data of the event. The nodes also forward the data of the event to a BS [2]. The BS forwards the data to a user through the existing communication infrastructure. Because the sensor networks operate in open environments such as a military environment, the sizes of the nodes are very small and unmanned [3,4]. Therefore, the nodes are captured and compromised easily by an attacker from the outside [2]. A false report injection attack especially causes a compromised node to generate a false report. Fig. 1 shows the operation of a false report injection attack. In Fig. 1, because of the false report generation of the compromised node in the sensor networks, the attacks lead to not only to false alarms, but also to the depletion of the limited energy of the sensor nodes, thus shortening the life of the networks [4]. In order to defend the false report injection attack, a lot of solutions have been proposed by many researchers [4-14]. Ye et al. proposed a solution which is called SEF [4]. In SEF, each intermediate node verifies an event report using authentication keys stochastically. The nodes forward the event report to the next node or drop it depending on the verification results. When the event report is false, the report is dropped. Thus, the false report is detected early by SEF. However, the number of authentication keys included in a node has to be large for higher verification of false report probability. If the number of keys of the node is more, then the energy consumption of the node is larger. The number of keys is not adjusted depending on the situation of the networks because it is fixed by the system. This means that it is difficult for the networks to be operated efficiently. In order to solve the problem, we proposed a method which adjusts the verification probability of the node using a scheme of a key sequence level. The key sequence level is an index in a hash chain of a key, which makes a message authentication code (MAC) in the event report. In the proposed method, the BS decides the key sequence level of an event report. Intermediate nodes receiving the event report verify it by comparing the key sequence level of the node's key with the level of a MAC in the event report. The key sequence level is settled by a fuzzy system in the BS. The fuzzy inputs determining the level are the average of the level of nodes in each path forwarding an event report, the density of neighbour nodes of a CoS, and the number of hop from the CoS to the BS. The proposed method includes security and efficient energy consumption of sensor nodes by determining the appropriate key sequence level. The rest of this paper is organized as follows. Section 2 describes the SEF. Section 3 explains the problem statements. Section 4 presents a system model of the proposed method and the operation process 
International Journal of Ambient Systems and Applications (IJASA) Vol.1, No.3,september 2013

of the proposed method. Section 5 shows the simulation results to evaluate the performance of the proposed method. Finally section 6 concludes this paper.

\section{BACKGROUND}

\subsection{Statistical en-route filtering}

SEF is a countermeasure method which can filter false reports early on that are made by a compromised node in a wireless sensor network using statistical verification during the en-route filtering phase. SEF consists of three phases: key assignment and report generation, en-route filtering, and sink verification. In the key assignment phase, before the sensor nodes are deployed in a sensor field, each node receives some keys where the number is fixed by the system randomly from a selected partition at random in the global key pool. In the report generation phase, after the nodes are deployed, sensing nodes detecting the event elect a CoS when an event occurs in the sensor network. They forward a partition index and a MAC to the BS which was generated by a key that each node includes to a BS. The CoS generates an event report with the event information and the received MACs and forwards the event report to the BS. In the en-route filtering phase, when an intermediate node receives the event report, it verifies the report stochastically. The node checks whether there are key indices of distinct partitions and MACs in the report. If there is either more than one key index or less than the one in the same partition or the number of them in the report does not correspond with the number of fixed MACs, the node regards the report as a false report and drops it. The node then examines whether there is a key index in the report corresponding with the key index of the node. If there is not a key index, the node forwards the report to the next node. If not, the node generates a MAC using its key. It then compares the MAC with the MAC in the report. If the MAC of the node is different from the MAC in the report, the report is regarded as a false report and is dropped. Otherwise, the report is regarded as a legitimate report and is forwarded to the next node. In the sink verification phase, all of the event reports arriving at the sink are verified, because the sink includes all keys in the global key pool. Thus, it can filter false reports out the false reports that are not filtered in the enroute filtering phase.

\section{Problem Statement}

If a false report injection attack occurs in a wireless sensor network, a compromised node will continuously generate many false reports, forwarding them to a BS. When the number of compromised nodes becomes larger, the number of false reports becomes larger. The sensor network which communicates many false reports may easily malfunction because of the energy depletion of the sensor nodes in the network. In SEF, a representative countermeasure method, in order to defend the network against the attack, intermediate nodes verify event reports using their assigned key before they are deployed in a sensor field. The key number is a very important element determining the verification probability of the report. The number of key that a node includes becomes larger, the probability becomes higher. However, the node has to consume a lot of verification energy. Moreover, when an attacker compromises a node, the number of keys that the attacker can get becomes larger. On the other hand, as the number of keys becomes smaller, the probability becomes smaller. However, the energy consumption used in the verification becomes lower. Subsequently, the number of keys that the attacker can get becomes smaller. Therefore, in order to efficiently operate the sensor network, it is important to trade energy consumption for security. Increasing the number of keys that the node includes is difficult in SEF because the energy of the sensor nodes is limited. To solve the problem, we use a key sequence level scheme. The scheme helps decrease the energy consumption and make up for security. Section 4 explains the proposed method using the key sequence level. 


\section{PROPOSED METHOD}

\subsection{System model and assumption}

A wireless sensor network consists of a BS and many sensor nodes. The BS includes a global key pool. In the global key pool, there are all the keys which are used in the sensor network. The BS also includes a fuzzy system that computes a key sequence level. The BS knows the average of the key sequence level of the intermediate nodes which forward event reports in each path, the density of neighbour nodes of the $\mathrm{CoS}$, and the number of hops from the $\mathrm{CoS}$ to the BS. The density of sensor nodes in the network is very high, and each node is small and each node includes simple computing capability and limited energy.

\subsection{Operation}

\subsubsection{Key assignment and report generation}

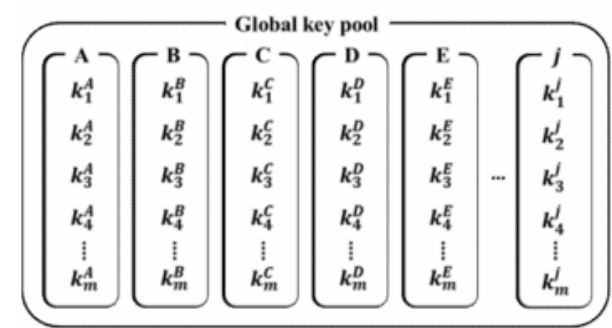

Fig. 2. A global key pool which consists of $\mathrm{j}$ hash chains

In Fig. 2, a hash chain is made up of m keys. The last sequence key in each hash chain $k_{m}^{j}$ is a seed key of each hash chain. The next sequence key is derived using a hash function with the seed key.

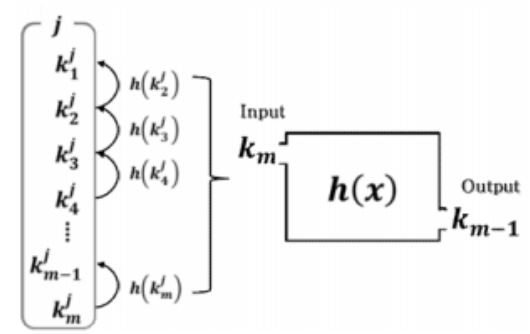

Fig. 3. Derivation of keys using the hash function

In Fig. 3, if the hash function receives a seed key as an input, the hash function outputs the derived key $k_{m}{ }_{1}$. If the hash function receives $k_{m} 1$ as an input, then it outputs a derived key $k_{m} 2_{2}$. This operation is repeated until the function outputs the key $k_{1}$. The derived keys are assigned to sensor nodes before the sensor nodes are deployed in the sensor network. 
International Journal of Ambient Systems and Applications (IJASA) Vol.1, No.3,september 2013

(a)

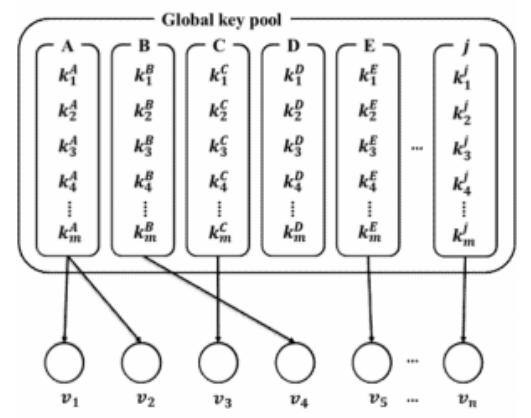

(b)

$$
\begin{aligned}
& v_{1} \text { key }=k_{46}^{A} \\
& v_{2} \text { key }=k_{4}^{A} \\
& v_{3} \text { key }=k_{19}^{C} \\
& v_{4} \text { key }=k_{27}^{B} \\
& v_{5} \text { key }=k_{38}^{E}
\end{aligned}
$$

Fig. 4. Example of an operation of key assignment in a global key pool (Fraction of $m=50$ )

Figure 4 (a) shows that just one key is randomly assigned to each sensor node $v_{1} \sim v_{n}$. Figure 4 (b) describes an example of the keys which are assigned to the sensor nodes. The sensor nodes derive other keys from the assigned key and a hash table. For example, In Fig. 4, a node $v_{3}$ receives a key $k_{19}^{C}$ in the $\mathrm{C}$ hash chain of the global key pool and then $v_{3}$ can get the keys from $k_{18}^{C}$ to $k_{1}^{C}$ using the hash function.

(a)

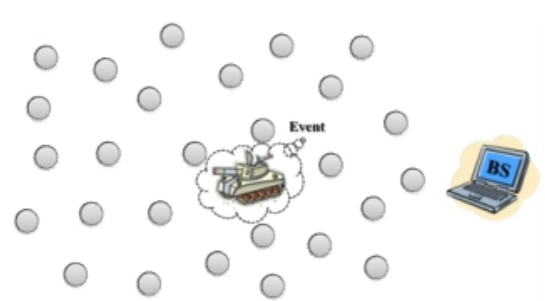

(c)

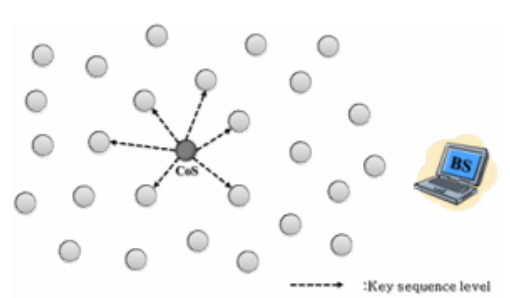

(b)

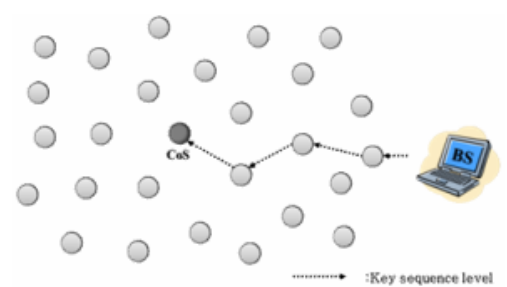

(d)

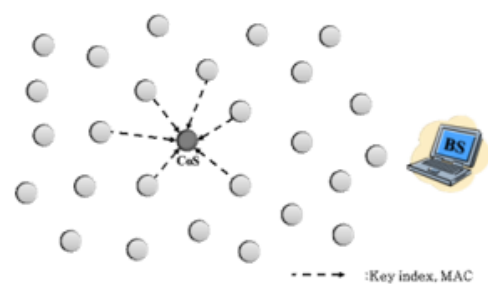

(e)

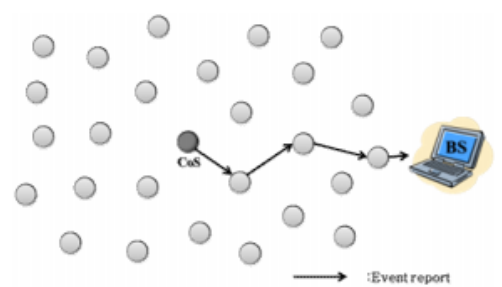

Fig. 5. The operation of a report generation

When an event occurs in the sensor field, multiple sensing nodes detect the event, as seen in Fig. 5 (a). The sensing nodes elect a CoS, which is a node that strongly detects the event. After the election of the CoS, the BS forwards the key sequence level, which is determined by a fuzzy system, to the CoS, as seen in Fig. 5 (b). The CoS forwards the key sequence level to its neighbour nodes in Fig. 5 (c). The neighbour nodes then generate MACs using the corresponding keys with information of the key sequence level from the $\mathrm{CoS}$ forwarding the MACs. A node 
which does not include the corresponding key with the key sequence level does not forward the MAC to the CoS, as seen in Fig. 5 (d). The CoS generates an event report using its information of the event and received MACs from the neighbour nodes. It then forwards the event report to the next node in Fig. 5 (e, f).

\subsubsection{The scheme of key sequence level}

The key sequence level is a generation index of a key, which makes a MAC in the event report in a hash chain. The key sequence levels of MACs in the event report are the same.

\subsubsection{Elements determining a key sequence level}

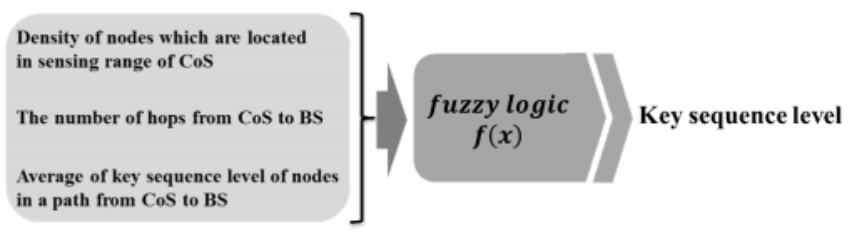

Fig. 6. Elements determining a key sequence level

In Fig. 6, the fuzzy system computes three input values in order to get the key sequence level as an output value. The three input values are the density of neighbor nodes, which are located within the sensing range of a $\mathrm{CoS}$, the number of hops from the $\mathrm{CoS}$ to a BS, and the average of the key sequence levels of intermediate nodes in a path.

\subsection{Density of neighbour nodes which are located in sensing range of a $\mathrm{CoS}$}

The higher the density of neighbor nodes in the CoS, the more the CoS collects MACs corresponding with the key sequence level. For example, let's suppose the key sequence level is 6 and the number of neighbor nodes in the sensing range of the CoS is 20 or 30 . In the case of 30 , the CoS collects more MACs corresponding to key sequence level 6 than in the case of 20. If the probability of the collection of MACs is higher, although the key sequence level is high, the CoS will collect enough MACs corresponding to the fixed number of MACs in the event report. Thus, the higher the density, the higher the key sequence level. On the other hand, the lower the density is, the lower the key sequence level is.

\subsection{The number of hops from a $\mathrm{CoS}$ to a $\mathrm{BS}$}

In order to decrease the energy consumption of the sensor nodes, false reports have to be filtered early. The more hops there are from the CoS to the BS, the more sensor nodes forward the event report, and a great amount of energy of the sensor nodes is consumed. Therefore, when the number of hops is large, the key sequence level has to be lower, and the report verification probability has to be high. On the contrary, when the number of hops is small, the key sequence level has to be high and the probability has to be lower.

\subsection{The average number of key sequence levels of intermediate nodes in a path}

The average number of key sequence levels of the forwarding node in each path is a very important element determining the key sequence level of the event report, because when the average of the key sequence level is high, the report verification probability becomes high, and 
when the average is lower, the probability becomes lower. Thus, the higher the average is, the higher the probability is. On the other hand, the lower the average is, the lower the probability is.

\subsubsection{Fuzzy membership function}

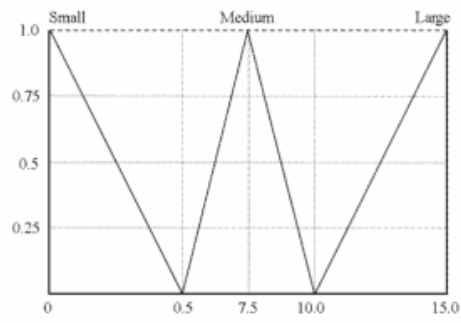

(a) The number of hops from the CoS to the BS

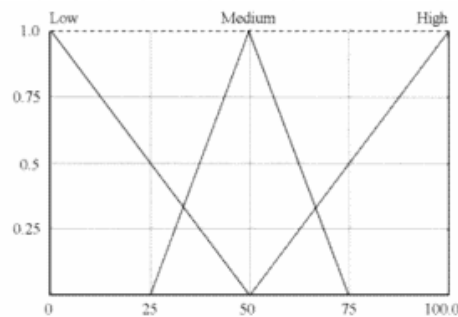

(c) The average of the key sequence level of intermediate nodes in each path

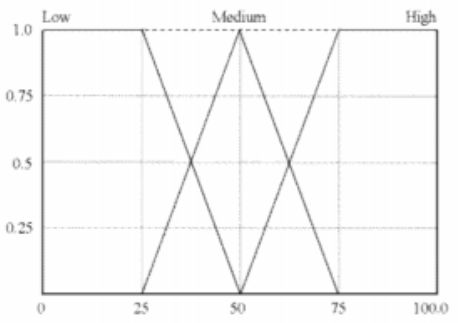

(b) Density of neighbor nodes in a sensing range of the $\mathrm{CoS}$

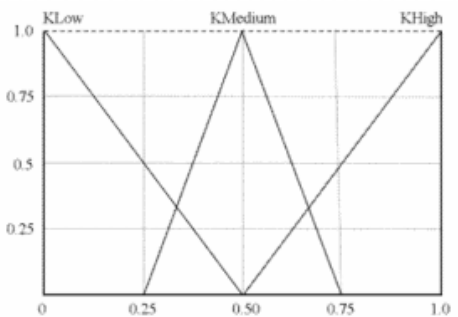

(d) Key sequence level of an event report

Fig. 7. Membership functions of input and output elements

In Fig. 7, (a) is a membership function of the number of hops from a CoS to the BS, (b) is the membership function of the density of neighbor nodes in the sensing range of the CoS, and (c) is a membership function of the average of the key sequence level of intermediate nodes in each path. In the membership function (a), fuzzy values are included in the fuzzy set which consists of three levels. The three levels are Low, Medium, and Large. In the membership functions (b) and (c), the fuzzy values are included in the fuzzy set. The fuzzy set consists of Low, Medium, and High. The membership function (d) is a membership function of a key sequence level of an event report. Its fuzzy values are included in a fuzzy set. The fuzzy set is composed of KLow, KMedium, and KHigh. In all fuzzy membership functions, the fuzzy values are in the range from $0-1$.

\subsubsection{Fuzzy rules}

Table 1. Fuzzy rules of the proposed method

\begin{tabular}{ccccc}
\hline \multirow{2}{*}{ No. } & \multicolumn{2}{c}{ INPUT } & OUTPUT \\
\cline { 2 - 5 } 0 & NUM_HOP & NEIGHBOR_DENSITY & AVERAGE_KEYLEVEL & KEY_LEVEL \\
\cline { 2 - 5 } & Small & High & High & KHigh \\
4 & Small & Medium & Medium & KHigh \\
8 & Small & Low & Low & KHigh \\
9 & Medium & High & High & KMedium \\
12 & Medium & Medium & High & KMedium \\
15 & Medium & Low & High & KMedium \\
20 & Large & High & Low & KLow \\
22 & Large & Medium & Medium & KLow \\
26 & Large & Low & Low & KLow \\
\hline
\end{tabular}


In Table1, NUM_HOP, NEIGHBORRR_DENSITY, and AVERAGE_KEYLEVEL are fuzzy inputs. NUM_HOP is the number of hops that an event report has to pass from a CoS to a BS. NEIGHBOR_DENSITY is density of neighbour nodes in a sensing range of the CoS. AVERAGE_KEYLEVEL is the average of the key sequence levels for the sensor nodes in each path. KEY_LEVEL is a fuzzy output and the key sequence level of the event report.

\subsubsection{En-route filtering}

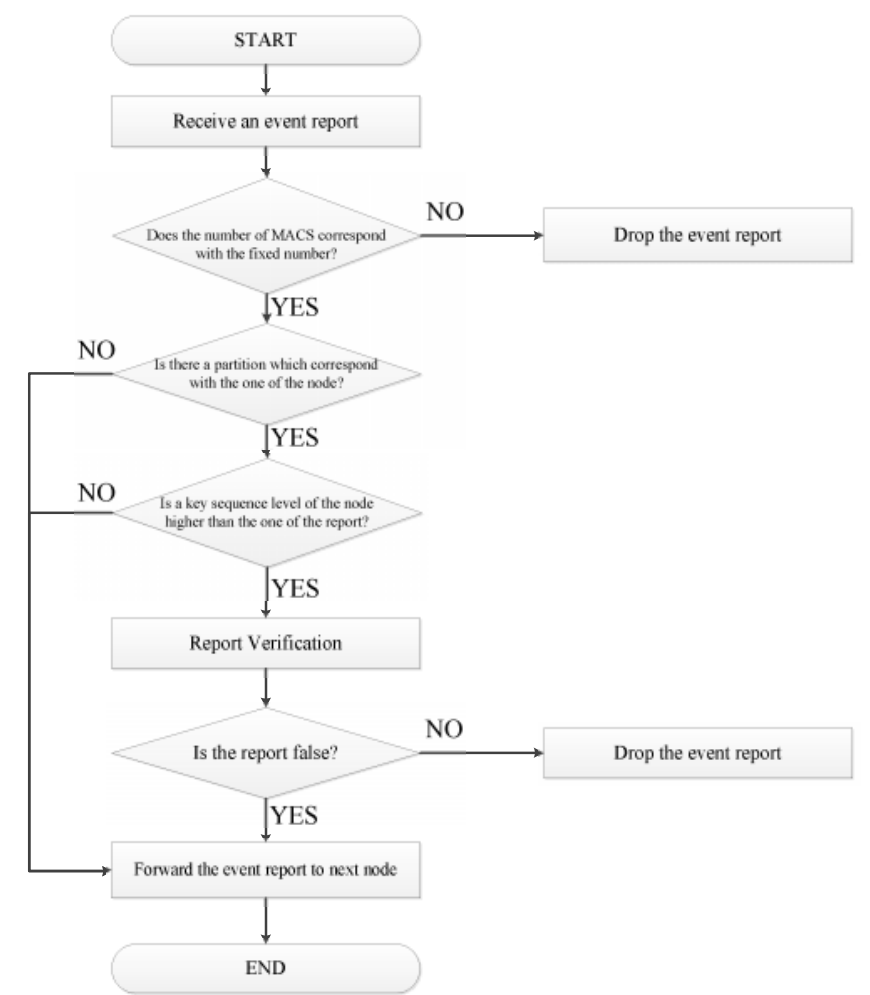

Fig. 8. A flow chart of an event report verification

Once the node receives the event report, it checks whether the number of MACs in the event report corresponds with the fixed number of MACs in the system (a). If the two number are different, the node regards the event report as a false report and drops the it (b). Otherwise, the node examines whether there is a corresponding partition in the event report with its partition of MACs (c). If there is not a partition, it forwards the event report to the next node (d). If there is a corresponding partition, the node compares the key sequence level of its key with the key sequence level of the MACs in the corresponding partition (e). When the key sequence level is lower than the one in the event report, it forwards the report to the next node (f). Alternatively, when the key sequence level is the same or higher than the one in the event report, it generates a MAC using its key and compares the MAC with the MAC of the event report (g, f). If the two MACs are the same, it regards the event report as a legitimate node and forwards the report to the next node (i). Otherwise, it regards the report as a false report and drops it (j).

\subsubsection{Base station verification}


When event reports arrive at a BS, the BS will verify all the MACs in the event reports because it includes a global key pool. If the BS receives an event report, it finds a key that corresponds to the key sequence level of a MAC in the event report. If there is a key, the BS generates a MAC using the key. It then compares the MAC with the MAC in the event report. If the two MACs are not the same, the BS drops the event report. Thus, although there are false reports which are not filtered in the en-route filtering phase, the BS verifies all false reports during this phase.

\section{Simulation}

In section 5, we compare the energy efficiency and security of our proposed method with that of the SEF in order to evaluate the performance of the proposed method. We evaluate the energy consumption of the sensor nodes versus the rate of false reports based on the number of compromised nodes among the sensing nodes, which generate MACs included in an event report, so as to compare the energy efficiency of the proposed method to that of the SEF. The number of false reports means arrivals at the BS which are not filtered in the en-route filtering phase. We also compared the number of false reports versus the rate of false reports in the proposed method to that of the SEF, so as to evaluate security.

Section 5.1 describes our simulation environment. Section 5.2 presents the simulation results.

\subsection{Simulation environment}

Table 2. A simulation environment

\begin{tabular}{lc}
\hline \multicolumn{1}{c}{ Content } & Values \\
\hline The number of the whole sensor nodes in a sensor network & 600 \\
The area of a sensor field & $100 \mathrm{~m} \mathrm{X} \mathrm{100m}$ \\
A sensing range of a sensor node & $10.0 \mathrm{~m}$ \\
Energy consumption per 1byte when a sensor node sends an event report & $16.25 \mathrm{~J}$ \\
Energy consumption per 1byte when a sensor node receives an event report & $12.5 \mathrm{~J}$ \\
Energy consumption of an event report verification of a sensor node & $75 \mathrm{~J}$ \\
The number of hash chains in a global key pool & 10 \\
The number of MACs in an event report & 5 \\
The number of occurring event reports in a sensor network & 100 \\
The number of keys in a hash chain & 50 \\
The number of keys included in a sensor node. & 25 \\
The packet size of an event report & $24 \mathrm{bytes}$ \\
\hline
\end{tabular}

\subsection{Simulation results}

In the simulation results, the rate of false reports is the entire number of event reports in the sensor field versus the number of false reports. The energy consumption is the sum total of the energy consumption of all of the sensor nodes in the sensor field.

Fig. 9 is a graph of energy consumption versus the rate of false reports in the proposed method and the SEF method when a sensing node which generates a MAC included in the event report is compromised. 


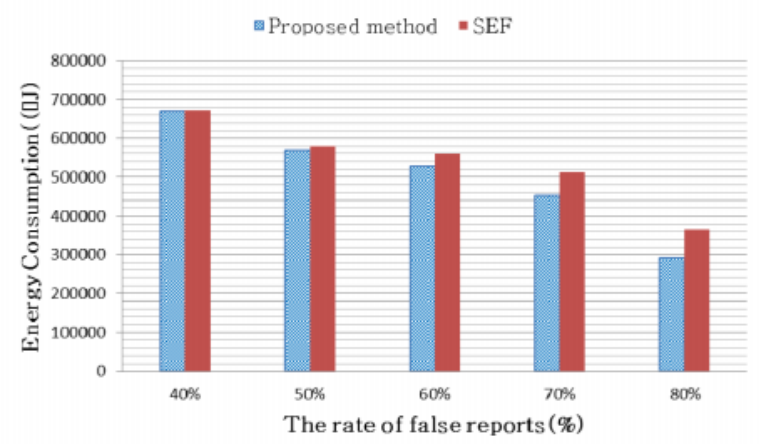

Fig. 9. Energy consumption versus the rate of false reports (Fraction of compromised node $=1$ )

In Fig. 9, we found that the proposed method consumes less energy of the sensor nodes than the SEF method. We also found that the higher the rate of false reports, the larger the gap between the energy consumption of the proposed method and SEF method. In the simulation result, the proposed method consumed an average of $7.89 \%$ less energy than the SEF.

Fig. 10 is a graph of the number of false reports arriving at the BS in the proposed method and the SEF method when a sensing node which generates a MAC included in the event report is compromised.

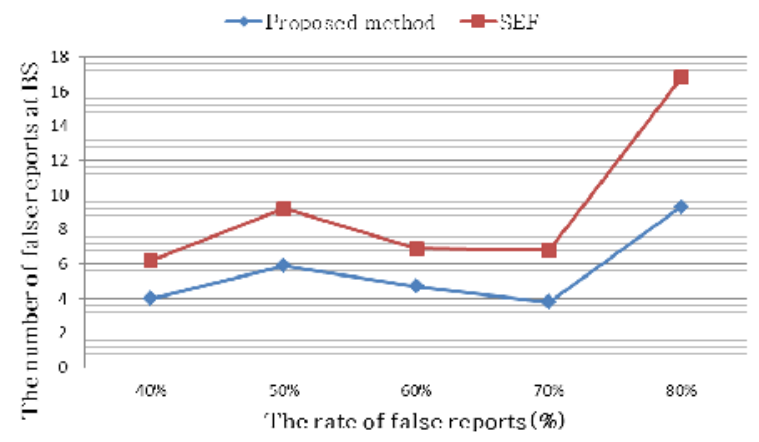

Fig. 10. The number of false report versus the rate of false reports (Fraction of compromised node $=1$ )

In Fig. 10, we found that the number false reports arriving at the BS in the proposed method is less than in the SEF method. In the simulation result, the number of false reports of the proposed method is an average of 3.64 less than in the SEF method.

Fig. 11 is a graph of energy consumption versus the rate of false reports in the proposed method and in the SEF method when the two sensing nodes which generate MACs included in the event report are compromised. 


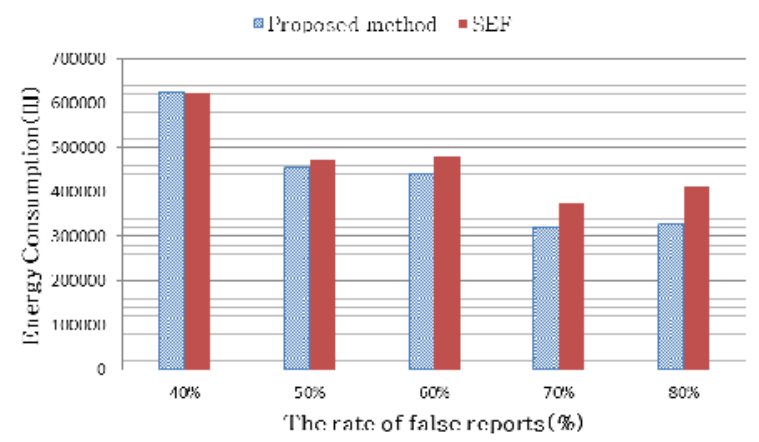

Fig. 11. Energy consumption versus the rate of false reports (Fraction of compromised nodes $=2$ )

In Fig. 11, the higher the rate of false reports is, the larger the gap between the energy consumption of the proposed method and that of the SEF method. We also know that the proposed method consumes less energy than the SEF. In the simulation result, the proposed method consumed an average of $9.09 \%$ less energy than the SEF.

Fig. 12 is a graph of the number of false reports arriving at the BS in the proposed method and that of the SEF method when two sensing node which generate MACs included in the event report are compromised.

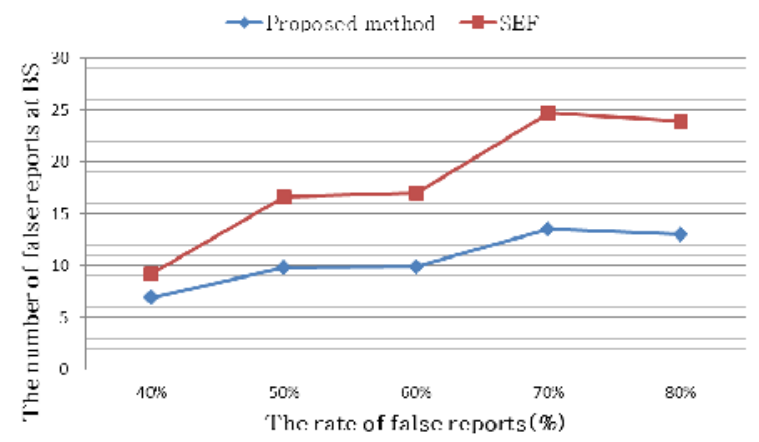

Fig. 12. The number of false reports versus the rate of false reports (Fraction of compromised nodes $=2$ )

In Fig. 12, we found that the number of false reports arriving at the BS in the proposed method is less than that of the SEF method when the two nodes are compromised. In the simulation results, the number of false reports is 7.66 less than that of the SEF method.

Fig. 13 is a graph of the energy consumption versus the rate of false reports in the proposed method and that of the SEF method when three sensing nodes which generate MACs included in the three event reports are compromised. 
International Journal of Ambient Systems and Applications (IJASA) Vol.1, No.3,september 2013

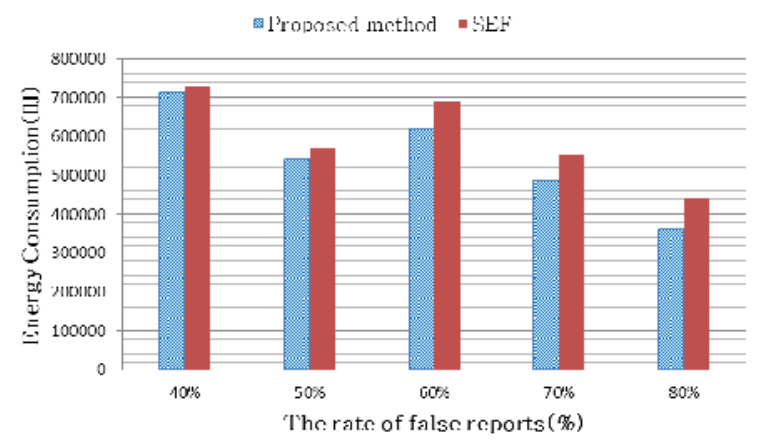

Fig. 13. Energy consumption versus the rate of false reports (Fraction of compromised nodes $=3$ )

In Fig. 13, the proposed method consumed less energy than the SEF. The higher the rate of false reports, the larger the gap of energy consumption is between the proposed method and the SEF method. In the simulation result, the proposed method consumed an average of $9.26 \%$ less energy than the SEF.

Fig. 14 is a graph of the number of false reports arriving at the BS in the proposed method and that of the SEF method when three nodes which generate MACs included in an event report are compromised.

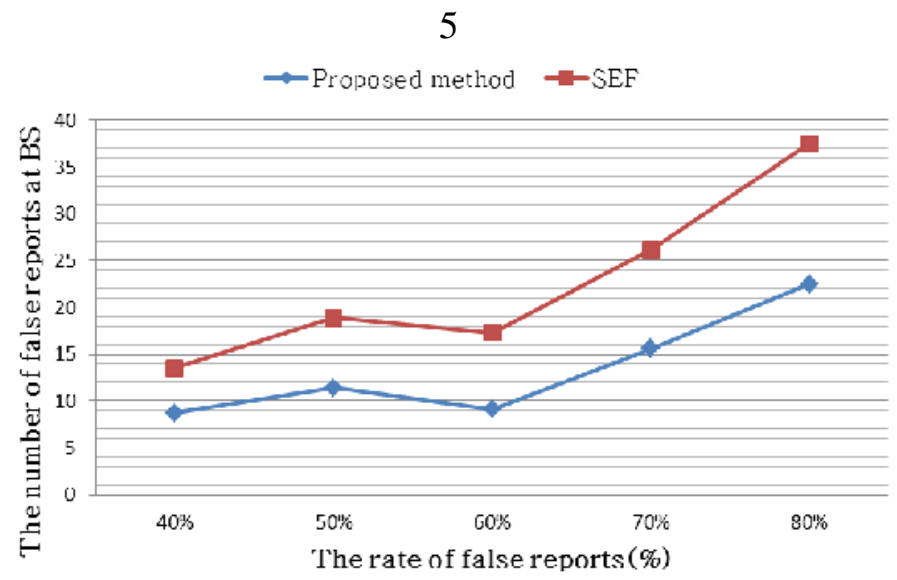

Fig. 14. The number of false reports versus the rate of false reports (Fraction of compromised nodes $=3$ )

In Fig. 14, we found that the number of false report arriving at the BS in the proposed method is less than that of the SEF method. In the simulation result, the number of false reports was 9.22 less than in the SEF.

Fig. 15 is a graph of the energy consumption versus the rate of false reports in the proposed method and in the SEF when four sensing nodes which generate MACs included in the three event reports are compromised. 


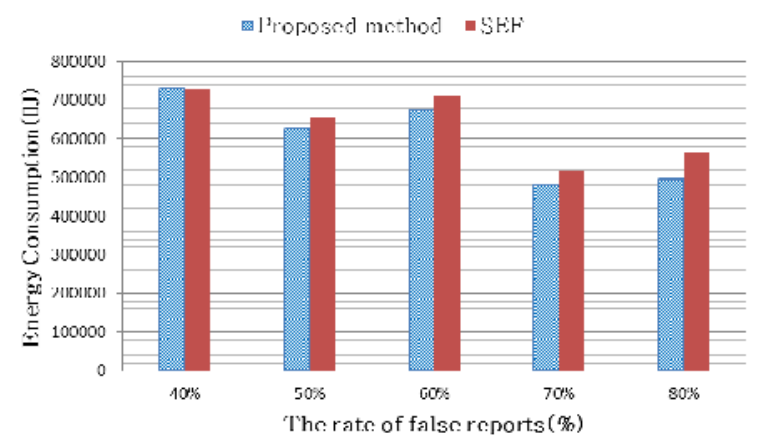

Fig. 15. Energy consumption versus the rate of false reports (Fraction of compromised nodes =4)

In Fig. 15, when the rate of false reports is above 50\%, the proposed method had better energy efficiency than the SEF. In the simulation result, the proposed method consumed an average $5.63 \%$ less energy than the SEF.

Fig. 16 is a graph of the number of false reports arriving at the BS in the proposed method and in the SEF when four nodes which generate MACs included in the event report are compromised.

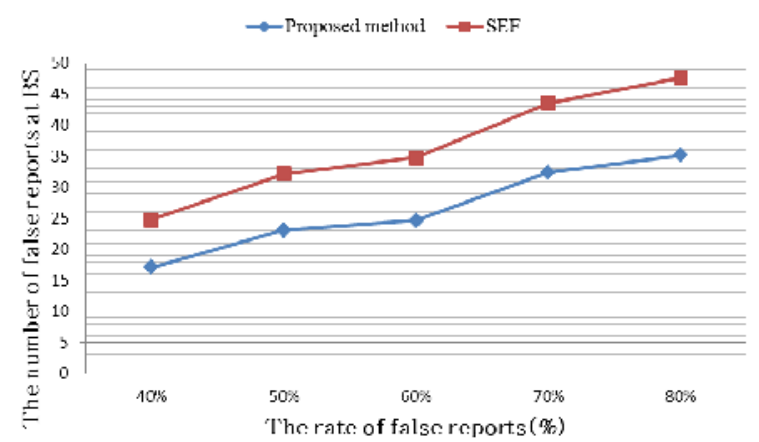

Fig. 16. The number of false reports versus the rate of false reports (Fraction of compromised nodes $=4$ )

In Fig. 16, we found that the number of false report arriving at the BS in the proposed method is less than in the SEF for all of the false reports rates. In the simulation result, the number of false reports was 10.14 less than that of the SEF method.

In the simulation results, the proposed method was an average of $7.9 \%$ better than the SEF with respect to energy efficiency. The number of false reports arriving at the BS in the proposed method was an average of 6.43 less than in the SEF method. We verified that the higher the rate of false reports in the sensor network, the better energy efficiency and security there was in the proposed method compared to the SEF method.

\section{CONCLuSiON}

In this paper, we proposed a solution which determines the key sequence level of MACs included in an event report and verifies them by comparing the key sequence level of the MACs in the event report with the key sequence level of a node which receives the event report. This is done in order to defend energy efficiency so when the probability of a false report in the event report is high, the event report verification probability is low, and the energy consumption of the sensor nodes is low. On the other hand, when the key sequence level is low, the event report verification probability is high, and energy consumption is high. Thus, it is important to determine an 
appropriate key sequence level. In order to determine the appropriate key sequence level, it is computed by a fuzzy system in a BS. Elements determining the key sequence level are the density of neighbor nodes in a sensing range of the CoS, the number of hops from the CoS to the BS, and an average of the key sequence level of intermediate nodes in each path.

We evaluated the energy efficiency and security of the proposed method by comparing it to the SEF method, which is a representative countermeasure method against the false report injection attack. We measured the energy consumption of the sensor nodes versus the rate of false reports in the sensor network to evaluate the energy efficiency of either the proposed method or the SEF. Additionally, we also measured the number of false report received in the BS versus the rate of false reports to evaluate the security of either the proposed method or the SEF. In the simulation results, the proposed method consumed an average of $7.9 \%$ less energy of the sensor network. Moreover the number of false reports arriving at the BS in the proposed method was an average 6.4 less than in the SEF method. It was found from the result that the proposed method has better energy efficiency and security than the SEF when the rate of false reports is high.

\section{ACKNOWLEDGEMENTS}

This research was supported by Basic Science Research Program through the National Research Foundation of Korea (NRF) funded by the Ministry of Education, Science and Technology (No. 2013R1A2A2A01013971)

\section{REFERENCES}

[1] I.F. Akyildiz, W. Su, Y. Sankarasubramaniam and E. Cayirci: Wireless sensor networks: a survey, Computer Networks, 38(4), 15 (2002) 393-422.

[2] Al-Karaki N., Kamal and A.E.: Routing techniques in wireless sensor networks: a survey, Wireless Communications, IEEE , 11(6), (2004) 6-28.

[3] Chris Karlof, David Wagner: Secure routing in wireless sensor networks: attacks and countermeasures, Ad Hoc Networks, 1, (2-3), (2003) 293-315.

[4] Fan Ye, Luo, H., Songwu Lu and Lixia Zhang: Statistical en-route filtering of injected false data in sensor networks, Selected Areas in Communications, IEEE Journal on , 23(4), (2005) 839- 850.

[5] Zhen Yu, Yong Guan: A dynamic en-route filtering scheme for data reporting in wireless sensor networks, IEEE/ACM, 18(1), (2010) 150-163.

[6] J. Hao Yang, Songwu Lu: Commutative cipher based en-route filtering in wireless sensor networks, Vehicular Technology Conference, 2004. VTC2004-Fall. 2004 IEEE 60th, vol. 2, (2004) 1223- 1227.

[7] Sencun Zhu, Setia, S., Jajodia, S. and Peng Ning: An interleaved hop-by-hop authentication scheme for filtering of injected false data in sensor networks, Security and Privacy, 2004. Proceedings. 2004 IEEE Symposium on , vol., no., (2004) 9-12, 259- 271.

[8] H.Y. Lee, T.H. Cho: Key Inheritance-Based False Data Filtering Scheme in Wireless Sensor Networks, Lect. Notes Comput. Sc, vol. 4371, Springer, Heidelberg (2006) 116-127.

[9] Zhang, W., Cao, G.: Group Rekeying for Filtering False Data in Sensor Networks: A Predistribution and Local Collaboration-based Approach, In: Proc. of INFORCOM, (2005) 503514.

[10] Perrig, A., Szewczyk, R. and Tygar, J.D., Wen, V., Culler, D.E.: SPINS: Security Protocols for Sensor Networks. Wirel. Netw. 8(5), (2002) 512-534.

[11] B.H. Kim, T.H. Cho: Condition-based Key Dissemination Period Determining Method in Sensor Networks, In Proc. Of ICACT, (2008). 
International Journal of Ambient Systems and Applications (IJASA) Vol.1, No.3,september 2013

[12] S.J. Lee, H.Y. Lee and T.H. Cho: A Threshold Determining Method for the Dynamic Filtering in Wireless Sensor Networks Based on Fuzzy Logic, International Journal of Computer Science and Network Security, 8(4), Apr. (2008).

[13] M.H Han, H. Y. Lee and T.H. Cho: Fuzzy-Based Verification-Probability Determination Method for Dynamic Filtering in Sensor Networks, International Journal of computer Science and Network Security, 8(8), Aug. (2008) 132-136.

[14] H.Y. Lee, T.H. Cho: Fuzzy Adaptive Selection of Filtering Schemes for Energy Saving in Sensor Networks, IEICE Transactions on Communications, E90-B(12), Dec. (2007) 3346-3353.

\section{Authors}

Hyum Woo Lee received his B.S. degrees in computer information from Kyungwon University, Korea, in February 2009 and M.S degrees in in Electrical and Computer Engineering from Sungkyunkwan University in 2013, respectively. Her research interests include wireless sensor network, security in wireless sensor networks, modelling \& simulation, and AI.

Su Man Nam received his B.S. degrees in computer information from Hanseo University, Korea, in February 2009 and M.S degrees in in Electrical and Computer Engineering from Sungkyunkwan University in 2013, respectively. He is currently a doctoral student in the College of Information and Communication Engineering at Sungkyunkwan University, Korea. His research interests include wireless sensor network, security in wireless sensor networks, and modelling \& simulation.
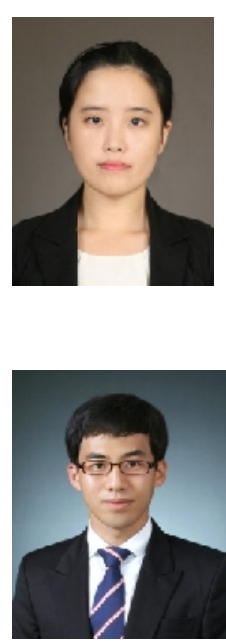

Tae Ho Cho received the Ph.D. degree in Electrical and Computer Engineering from the University of Arizona, USA, in 1993, and the B.S. and M.S. degrees in Electrical Engineering from Sungkyunkwan University, Republic of Korea, and the University of Alabama, USA, respectively. He is currently a Professor in the College of Information and Communication Engineering, Sungkyunkwan University, Korea. His research interests are in the areas of wireless sensor network, intelligent systems, modeling \& simulation, and enterprise resource planning.

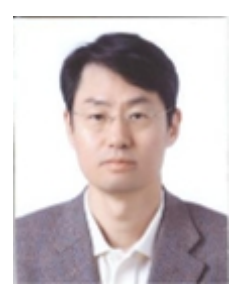

